\title{
1 Basic Properties of Diamond: Phonon Spectra, Thermal Properties, Band Structure
}

\section{GORDON DAVIES}

Physics Department, King's College London, UK

1.1 Introduction 3

1.2 The static lattice 4

1.2.1 Dimensions of the lattice 4

1.2.2 Thermal expansion of the lattice 5

1.2.3 Isotope effects on the dimensions of the lattice 6

$\begin{array}{ll}1.2 .4 \text { Elastic constants } & 7\end{array}$

1.3 Vibrations 9

1.3.1 Dispersion curves and the density of phonon states 10

1.3.2 Special values of $\mathbf{k}$ : the Raman frequency 11

1.3.3 Special values of $\mathbf{k}$ : other modes 13

1.3.4 Two- and three-phonon processes 14

$\begin{array}{ll}1.3 .5 \text { Isotope effects } & 16\end{array}$

$\begin{array}{ll}\text { 1.3.6 Specific heat } & 17\end{array}$

1.3.7 Thermal conductivity 18

$\begin{array}{ll}1.4 \text { Electronic energy states } & 20\end{array}$

1.4.1 Dispersion curves 20

1.4.2 Temperature dependence and isotope dependence of the lowest energy gap 22

1.4.3 Exciton states and electron-hole condensates 23

$\begin{array}{ll}1.4 .4 \text { Dielectric properties } & 24\end{array}$

1.5 Summary 25

References $\quad 25$

\subsection{INTRODUCTION}

Diamond has been known for over two thousand years. For most of that time it was only valued for its appearance and for its mechanical properties. With the rapid increase in the control of its synthesis, new possibilities have arisen to exploit its electrical and 
optical properties. Diamond has the largest electronic energy gap among the elemental semiconductors, and one of the largest known energy gaps of all semiconductors. It has high diffusion energies for impurities and defects, and stable surfaces. The combination of light atomic mass and strong chemical bonding results in diamond having relatively high vibrational frequencies compared with other semiconductors. Related to this, at room temperature and above, the ability of diamond to conduct heat from an active area is unusually high. These properties should allow diamond to be used increasingly in a range of applications, from surface-mediated processes to high-power devices [1]. Developments of the use of diamond require knowledge of a very wide range of properties. Some have been known for many years, such as the thermal expansion of diamond, which is required to make a heterogeneous structure of diamond and another material. Other properties have only been mapped out recently. For example, to obtain complete control of the lattice we need to know the effects not only of impurities but also of the disorder from the isotope content of the lattice. In yet other areas, exploiting high speed (terahertz) or small dimensions (nanometre), there is still much to learn.

In this chapter, our current understanding of the basic properties of diamond is outlined, focusing primarily on the experimental data.

\subsection{THE STATIC LATTICE}

The carbon in a natural diamond has two stable isotopes, ${ }^{12} \mathrm{C}$ in $98.9 \%$ natural abundance, and ${ }^{13} \mathrm{C}$ at $1.1 \%$ abundance. The nuclear spins are respectively zero and one-half. The simple chemical nature of diamond allowed its chemistry to be determined very early on [2], while the high crystalline quality of many natural diamonds permitted the crystal structure of diamond to be determined in the pioneering X-ray diffraction studies [3].

Diamond crystallises in a face-centred cubic lattice structure with a cube edge length denoted by $a_{0}$. Each lattice site in the face-centred cubic lattice is populated by two atoms, separated by $\sqrt{3 / 4} a_{0}$, approximately equal to $0.154 \mathrm{~nm}$. The interatomic spacing is small compared with other semiconductors (compare the more typical $0.234 \mathrm{~nm}$ for silicon).

\subsubsection{Dimensions of the lattice}

A critical discussion of precision X-ray diffraction measurements on natural diamonds suggests that the cube edge length $a_{0}$ of pure diamond, measured at room temperature (approximately $20^{\circ} \mathrm{C}$ ), with natural isotope content, is [4] given by:

$$
a_{0}=0.356710(4) \mathrm{nm} \text {. }
$$

The actual value for a particular diamond will depend on its impurity level, its isotope content and its temperature. To give a feeling of scale, a change in $a_{0}$ by 1 part in $10^{5}$ would result if the temperature of the sample were to change by $12{ }^{\circ} \mathrm{C}$, or if $7 \%$ of the atoms were ${ }^{13} \mathrm{C}$, or if 0.03 atomic-percent of the atoms were nitrogen atoms in the common pair ('A') form. Specifically, Lang and Pang [4] estimate that an atomic fraction 
$c_{\mathrm{N}(\mathrm{A})}$ of nitrogen in the $\mathrm{A}$ form will produce a lattice expansion of:

$$
\frac{\Delta a_{0}}{a_{0}}=0.036 c_{\mathrm{N}(\mathrm{A})}
$$

For nitrogen in the single-substitutional form, commonly found in as-grown synthetic diamonds, they estimate that an atomic fraction $c_{\mathrm{N}(\mathrm{C})}$ of nitrogen in this dispersed (' $\mathrm{C}$ ') form produces what can be a very significant effect:

$$
\frac{\Delta a_{0}}{a_{0}} \sim 0.1 c_{\mathrm{N}(\mathrm{C})} .
$$

The effect of pressure on the lattice is discussed in Section 1.2.4.

\subsubsection{Thermal expansion of the lattice}

Atomic vibrations in diamond are not precisely harmonic. For a typical chemical bond we expect the energy required to compress it by a small amount to be greater than the energy to lengthen it by the same amount. As the atoms vibrate, they can lengthen the bond more easily than compressing it. With increasing temperature, the vibrational amplitude increases, and the asymmetry becomes more pronounced. The lattice expands with increasing temperature, as a result of the anharmonicity in the vibrations.

In diamond, the cube edge length $a_{0}$ increases monotonically as the temperature increases. Recommended values of the coefficient of linear expansion $(\alpha)$ have been compiled by Slack and Bartram [5] based on work on natural diamonds. Up to a temperature of $500 \mathrm{~K}, \alpha$ may be accurately parameterised as a function of the temperature $T$ in kelvin by:

$$
\alpha=1.08 \times 10^{-11} T^{2},
$$

such that $a_{0}$ increases as the cube of $T$; the expansion (caused by vibrational anharmonicity) is proportional to the specific heat (resulting from the harmonic vibrations, see Section 1.3.6). With increasing $T, \alpha$ is expected to saturate to a constant value, and above $500 \mathrm{~K}$ a good parameterisation is:

$$
\alpha=7.95 \times 10^{-6} \times(1-\exp [-T / 1220]) .
$$

Wild and Koidl [6] have reported measurements of the expansion of polycrystalline, chemical-vapour-deposited (CVD) diamond, from 20 to $500^{\circ} \mathrm{C}$. Their data are a few $(<5)$ percentage points higher than the recommended values of Slack and Bartram [5]. Wild and Koidl [6] describe their data using the temperature $t$ in ${ }^{\circ} \mathrm{C}$ as:

$\alpha=8.19 \times 10^{-7}+1.107 \times 10^{-8} t-1.48 \times 10^{-11} t^{2}+1.08 \times 10^{-14} t^{3}, \quad 20<t<500$.

The coefficient $\alpha$ of lattice expansion has been accurately calculated up to $400 \mathrm{~K}$ in the $a b$ initio model of lattice vibrations of Pavone et al. [7]. The coefficient has very similar values to those of other semiconductors when the temperature is scaled [5] by 
an effective Debye temperature ( $\sim 2240 \mathrm{~K}$ for diamond), but $\alpha$ is always positive for diamond in contrast to cubic silicon and germanium, where the lattice initially contracts (e.g. for $T<120 \mathrm{~K}$ for silicon).

\subsubsection{Isotope effects on the dimensions of the lattice}

The cube edge length $a_{0}$ has been measured in a series of diamonds synthesised at high pressure and high temperature, with five different atomic fractions $x$ of ${ }^{13} \mathrm{C}$, the remainder being ${ }^{12} \mathrm{C}$. The variation in $a_{0}$ was found to be linear in $x$, following [8]:

$$
a_{0}=0.356714-5.4 \times 10^{-5} x \mathrm{~nm} .
$$

The data are for $25^{\circ} \mathrm{C}$, and the value for $x=0$ is in agreement with Equation (1.1). The fractional change for complete substitution, at $x=1$, is then given by:

$$
\frac{\Delta a_{0}}{a_{0}}=-1.5 \times 10^{-4} .
$$

In contrast, Yamanaka et al. [9] have reported a nonlinear variation with $x$,

$$
a_{0}=0.356712-9.0 \times 10^{-5} x+3.7 \times 10^{-5} x^{2} \mathrm{~nm} .
$$

The change in the volume is another consequence of the anharmonicity of the vibrations and may be calculated ab initio [10]. The physical basis is as follows. If we imagine a diamond made of ${ }^{12} \mathrm{C}$ that is at $0 \mathrm{~K}$, and the atoms are static (there is no zero-point motion), then the chemical bonding will result in a certain inter-atomic spacing as a result of minimising the energy. We now allow the atoms to vibrate in their zero-point motion. In the harmonic approximation, the zero point motion of the $i$ th mode is $\frac{1}{2} \hbar \omega_{i}$. All the modes therefore increase the energy by $\frac{1}{2} \sum_{i} \hbar \omega_{i}$. However, the modes are not strictly harmonic, and a change (usually an increase) in the bond length lowers the zero-point energy (Section 1.3.3). To minimise the vibrational energy, the lattice expands, but that expansion stretches the chemical bonds, and so increases the elastic energy of the lattice. A balance is reached where the decrease in vibrational energy is balanced by the increase in elastic energy. If we now repeat the argument for a ${ }^{13} \mathrm{C}$ diamond, the same processes occur, but now the vibrational frequencies are all smaller by a factor of $\sqrt{12 / 13}$, and so the zero-point energy is smaller. Expansion produces a smaller reduction in energy (because each $\hbar \omega_{i}$ is smaller), and so there is less energy to be saved by an expansion - starting from the imaginary static lattice, a ${ }^{13} \mathrm{C}$ diamond would not expand as much as a ${ }^{12} \mathrm{C}$ diamond. The value of $a_{0}$ is therefore smaller for a ${ }^{13} \mathrm{C}$ diamond than for a ${ }^{12} \mathrm{C}$ diamond. To calculate the effect, we need to know the change in frequency of each mode with the bond-length, how many modes we have at each frequency, and the elastic constants of diamond. The first is not well known (Section 3.3), the second and third are known (Sections 3.1 and 2.4). This argument applies at low temperatures (or at room temperature for diamond). In the limit of high temperature, the energy in each vibrational mode becomes the Boltzmann value of $k_{\mathrm{B}} T$, which is independent of the frequency of the mode, and so is independent of its mass. At a very high temperature, a ${ }^{13} \mathrm{C}$ diamond would have the same lattice spacing as a ${ }^{12} \mathrm{C}$ diamond. 
The content of ${ }^{13} \mathrm{C}$ in natural diamonds is slightly variable, with changes in ${ }^{13} \mathrm{C}$ of a few parts per thousand being observed. The variation is significant in geological studies but not likely to be of concern here.

\subsubsection{Elastic constants}

Diamond is well known to have large elastic constants. Because diamond has a facecentred cubic lattice, we can define Cartesian coordinates $x, y, z$ with respect to the lattice, and the repeat distance along $x, y$ or $z$ is $a_{0}$. For a macroscopic diamond, any physical property measured along one of the cubic axes will have the same value along either of the others. This result produces a major simplification in the elastic constants: for a cubic crystal, there are only three elastic constants. We denote a strain generally by $e$. If we compress the crystal along the $x$ axis, the fractional change in length along the $x$ axis, the strain, is $e_{x x}$. This compression will cause the crystal to expand in the $y$ and $z$ directions, through the 'Poisson's ratio' effect, producing strains $e_{y y}$ and $e_{z z}$ in those directions. The stress, $s$, required to produce this particular deformation is given by the force acting in the $x$ direction, on an area whose normal is in the $x$ direction, and so is denoted $s_{x x}$. The relationship between the stress and the strains is given in terms of the elastic constants $c_{11}$ and $c_{12}$ as:

$$
s_{x x}=c_{11} e_{x x}+c_{12} e_{y y}+c_{12} e_{z z} .
$$

A different type of distortion is a shear, for example, when a cube face normal to $y$ is sheared in the $z$ direction to give a strain $e_{y z}$. To achieve this strain requires a shear stress, which is a force acting in the $z$ direction on a unit-area face whose normal is in the $y$ direction:

$$
s_{y z}=c_{44} e_{y z}
$$

The numerical subscripts denote the combinations $1=x x, 2=y y, 3=z z, 4=y z, 5=$ $z x, 6=x y$. Equation (1.10) may be rewritten with a cyclic permutation of $x, y$ and $z$, but using the same elastic constants (i.e. for the cubic crystals, $c_{11}=c_{22}=c_{33}$ ). Similarly, Equation (1.11) may be written for $z x$ and $x y$ shears, and $c_{44}=c_{55}=c_{66}$ ).

Brillouin scattering provides one method of measuring the elastic constants. It occurs when a photon is scattered into a new direction in the crystal, with the wave vector being conserved by emitting a phonon. The wave vector of the phonon is therefore known, and the change in energy of the scattered photon gives the frequency of the phonon. Hence the speed of the phonon (the speed of that sound wave) is known, and the speed of sound is linked to the elastic constants. The values at room temperature, for natural isotope abundance diamond, have been measured by Brillouin scattering [11]:

$$
c_{11}=1080.4 \pm 0.5, c_{12}=127.0 \pm 0.7, c_{44}=576.6 \pm 0.7 \mathrm{GPa}
$$

The 'bulk modulus' $K(=V \mathrm{~d} P / \mathrm{d} V)$ is then given by:

$$
K=\left(c_{11}+2 c_{12}\right) / 3=444.8 \mathrm{GPa} \text {. }
$$


With increasing temperature from $300 \mathrm{~K}$, each elastic constant $c$ decreases from its value $c_{0}$ given immediately above, the change depending on the square of the temperature. The effect may be parameterised by:

$$
c=c_{0}+k\left(T^{2}-9.0 \times 10^{4}\right)
$$

where the term $k$ is the best-fitting parameter to the data, and $T$ is in kelvin. For $c_{11}$, the value of $k$ is $(-29 \pm 8) \times 10^{-6}$, for $c_{12}, k=(-3 \pm 18) \times 10^{-6}$, and for $c_{44}, k=(-22 \pm$ 7) $\times 10^{-6} \mathrm{GPa} \mathrm{K}^{-2}[12]$.

Brillouin scattering measurements have been reported by Jiang et al. [13] for CVD diamond films, oriented with a $\langle 110\rangle$ direction normal to the surface of the sample, giving results, within their uncertainty, of the values in Equation (1.12). If the size of the diamond crystal is not macroscopic in all dimensions, the elastic constants would be expected to remain at their bulk values until the crystal shrinks to a few atoms in size. However, the wavelengths of the sound waves are $\sim 100 \mathrm{~nm}$, illustrating that the point when a crystal stops being macroscopic is dependent on the technique in use.

Macroscopic (beam-bending) measurements of the elastic properties of polycrystalline diamond have been reported by Szuecs et al. [14]. In many applications it is convenient to be able to calculate the effect of stress by using a simple 'Young's modulus' $E$ which is defined in terms of the applied stress $s$ and the applied strain $e$ as $E=s / e$. However, the elastic properties are not isotropic. If the diamond is compressed by a stress $s$ acting directly along the $x$ axis, so that $s_{x x}=s$ and all other $s_{i j}=0$, then the strains are known from the three equations like Equation (1.10), and Young's modulus is:

$$
E_{001}=\left(c_{11}^{2}+c_{11} c_{12}-2 c_{12}^{2}\right) /\left(c_{11}+c_{12}\right) .
$$

In contrast, a stress $s$ acting directly down a $\langle 111\rangle$ axis has stress components $s_{i j}=s / 3$ for all $i, j$, and

$$
E_{111}=3 c_{44}\left(c_{11}+2 c_{12}\right) /\left(c_{11}+2 c_{12}+c_{44}\right) \text {. }
$$

These values are, respectively, the minimum and maximum values of $E$ for diamond. The values in Equation (1.12) give $E_{001}=1054 \mathrm{GPa}$ and $E_{111}=1208 \mathrm{GPa}$, a variation of $\sim 10 \%$ about the mean. A polycrystalline sample would be expected to have a Young's modulus in this range, if the elastic properties of the material are not determined by the grain boundaries, a condition readily achieved in current CVD diamond: Szuecs et al. [14] report values for $E$ at room temperature of $1143 \mathrm{GPa}$, with a $2.5 \%$ scatter. The value is irreversibly reduced by degradation of the material when it is heated above 1000 $\mathrm{K}\left(700^{\circ} \mathrm{C}\right)$ for the best quality, optical-grade, CVD diamond used. Below this transition point, they reported that Young's modulus for CVD diamond decreases with temperature $T$ (in K) over the range 300 to $1000 \mathrm{~K}$, at a sample-dependent rate that was significantly higher than that reported for single-crystal diamond (Equation 1.14). The rate was fitted by a linear dependence [14]:

$$
E(T)=E(300)[1+k(T-300)], k \sim-1 \times 10^{-4} \mathrm{~K}^{-1} .
$$

Data at high pressure are important, for example, when diamond is used in a high-pressure anvil cell. Occelli et al. [15] have measured the lattice size, using X-ray diffraction, up to 
$140 \mathrm{GPa}$ as a function of pressure $P$ (in GPa, determined from the ruby pressure scale). They find that $P$ and the molecular volume $V\left(\mathrm{in}^{3} \mathrm{~mol}^{-1}\right)$ accurately follow the Vinet equation:

$$
P=3 K_{0}(1-X) X^{-2} \exp \left[3\left(K_{0}^{\prime}-1\right)((1-X) / 2]\right.
$$

where $X=\left(V / V_{0}\right)^{1 / 3}$. The subscript 0 indicates values at atmospheric pressure, and $K_{0}^{\prime}$ is the pressure derivative of the bulk modulus. The experimental value for the constants, valid up to a stated $140 \mathrm{GPa}$, were $V_{0}=3.4170(8) \mathrm{cm}^{3} \mathrm{~mol}^{-1}, K_{0}=446(1) \mathrm{GPa}$, essentially as in Equations (1.1) and (1.13), and $K_{0}^{\prime}=3.0(1)$. However, the pressure calibration has been criticised; theoretical calculations predict [16]:

$$
K_{0}^{\prime}=3.72 \text { to } 3.8 \text {. }
$$

We have seen that the lattice spacing for ${ }^{13} \mathrm{C}$ diamond is smaller than for ${ }^{12} \mathrm{C}$ diamond (Section 2.3). There is a corresponding increase in the elastic constants. Ramdas et al. [17] report that for ${ }^{13} \mathrm{C}$ diamond at room temperature the following holds:

$$
c_{11}=1083.3 \pm 0.5,\left(c_{11}+2 c_{12}+4 c_{44}\right) / 3=1218.7 \pm 0.5 \mathrm{GPa} .
$$

The value of the second term in ${ }^{12} \mathrm{C}$ diamond is $1214 \mathrm{GPa}$ from Equation (1.12). Ramdas et al. [17] suggest that ${ }^{13} \mathrm{C}$ diamond is arguably the hardest known material.

The combination of the small interatomic distance and the high elastic constants results, qualitatively, in very high strain energies when most impurity atoms are placed into diamond, causing natural diamonds to be surprisingly pure.

\subsection{VIBRATIONS}

The vibrational dispersion curves, plotting the frequencies $(\omega)$ of vibrations against their wavevector $(\mathbf{k})$, are well established for most semiconductor materials. For silicon and germanium, the curves have very similar shapes and may be closely superimposed after linear scaling. The dispersion curves of diamond are distinctly different. The acoustic modes in $\mathrm{Si}$ and $\mathrm{Ge}$ have long regions near the zone boundaries in which $\omega$ varies little with $\mathbf{k}$, resulting in pronounced peaks in the phonon densities of states for the acoustic modes; diamond does not show this behaviour. Further, in contrast to $\mathrm{Si}$ and Ge, the maximum vibrational frequency is not at the zone centre.

Even at the limit of low temperature, atomic vibrations are occuring in a crystal as a result of zero-point motion. Consequently, all processes in a crystal involve the vibrations of the lattice. For example, when, at $0 \mathrm{~K}$, an electron is excited between two 'electronic' states $\mathrm{A}$ and $\mathrm{B}$; the process involves taking the crystal from the zero-point vibrational level with the electron in state A to the zero-point vibrational level with the electron in state B. Because the electronic state has changed, those vibrational states may not be identical. Some effects are small: at $0 \mathrm{~K}$, it is estimated that the zero-point motion in the anharmonic potential results in the lattice parameter $a_{0}$ being about $0.5 \%$ larger than would be the case for a static lattice [18]. More obviously, the temperature dependence of the lattice size (Section 1.2.2) and of the elastic constants (Section 1.2.4) arises primarily 
through the population of lattice modes (assuming a lightly doped or pure diamond, with negligible electronic excitation).

A harmonic vibration of angular frequency $\omega$ has energy levels $\left(n+\frac{1}{2}\right) \hbar \omega$ and a Bose Einstein population at temperature $T$ given by:

$$
\bar{n}=1.0 /\left[\exp \left(\hbar \omega / k_{\mathrm{B}} T\right)-1\right]
$$

$\bar{n}$ has the physical meaning that it is the mean value of $n$, the mean quantum state occupied. At high $T, k_{\mathrm{B}} T>\hbar \omega$, the population of a mode becomes $\bar{n}=k_{\mathrm{B}} T / \hbar \omega$, and the energy in that mode is $k_{\mathrm{B}} T$, which is independent of the frequency of the mode. Thermally induced effects therefore tend to be linear in $T$ at high $T$. At the limit of low temperature, all vibrational states are populated in their zero-point level, $\frac{1}{2} \hbar \omega$, which is independent of $T$. The difference between the value of a property observed as $T \rightarrow 0$, and the value extrapolated to $T=0$ from the high-temperature values is frequently referred to as the zero-point renormalisation, and controls, for example, much of the isotope dependence of the property.

When a transition involves the creation or destruction of a phonon, the probabilities, $P_{e}$ and $P_{a}$, of emitting or absorbing a phonon of frequency $\omega$ depend on temperature through:

$$
P_{e} \propto[1+\bar{n}], P_{a} \propto \bar{n}
$$

At low $T$, only states of small $\omega$ can be populated, any thermal effect is weighted towards the vibrations of low frequency, and the high frequency vibrations are irrelevant. In diamond, the maximum lattice frequency of $1335 \mathrm{~cm}^{-1}$ has an energy quantum $\hbar \omega$ equal to $k_{\mathrm{B}} T$ at $1900 \mathrm{~K}$, requiring the use of high temperatures if all the vibrational modes are to be thermally populated.

To understand the vibrational properties of diamond we need to know the values of the frequencies and how many modes of vibration we have in each small frequency interval.

\subsubsection{Dispersion curves and the density of phonon states}

As expected for a simple crystal, the vibrational dispersion curves of diamond were among the first to be measured by neutron scattering [19]. However, these data were limited to room temperature and pressure, and some key data were sparse: for example, only one measurement was made for the highest frequency optic modes with $\mathbf{k} \|\langle 001\rangle$. Subsequently, it was observed that the two-phonon Raman spectrum (Section 1.3.3) showed a peak at a frequency shift about $3 \mathrm{~cm}^{-1}$ greater than twice the Raman frequency [20-23], as a result of the vibrational frequency for the highest energy ('longitudinal optic') modes increasing slightly in frequency as $\mathbf{k}$ moves away from the zone centre. That maximum has been confirmed by inelastic X-ray scattering measurements for $\mathbf{k} \|\langle 001\rangle$ [24]. Burkel et al. [25] have also reported on the dispersion curve of the longitudinal acoustic mode along $\langle 001\rangle$, confirming the neutron data. The increase in frequency in the dispersion curves for the optic mode on moving away from the zone centre is a result of the unusually strong next-nearest-neighbour force interactions in diamond compared to other zinc blende and elemental semiconductors [7]. 


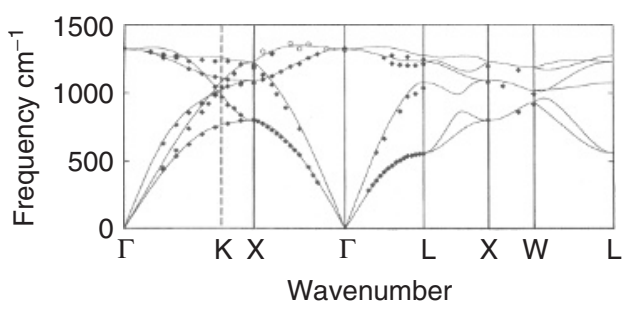

Figure 1.1 Phonon dispersion curves calculated by Pavone et al. [7], for phonon wavevectors $\mathbf{k}$ parallel to the major crystallographic axes. Point $\Gamma$ has $\mathbf{k}=0$, and $\mathrm{X}, \mathrm{L}$ and $\mathrm{K}$ are respectively at the $\langle 001\rangle,\langle 111\rangle$ and $\langle 110\rangle$ zone boundaries. The filled circles are neutron scattering data [19] and the open circles are X-ray scattering data [24], at room temperature

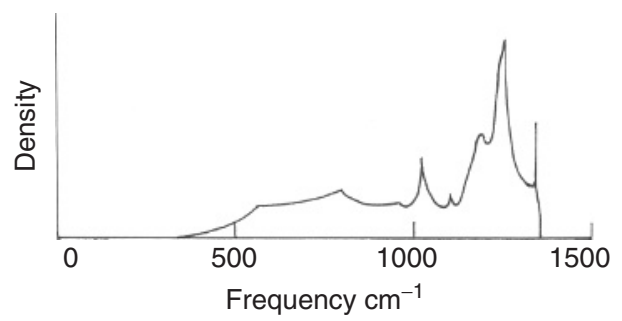

Figure 1.2 The phonon density of states, calculated by Pavone et al. [7], for diamond at room temperature

The measured dispersion curves provide a check for calculated dispersion curves, now available at high accuracy (e.g. Refs [7, 26], and Figure 1.1). Calculations of the vibrational frequencies allow, effectively, interpolation between the measured data, so that the number of modes of vibration $g(\omega) \mathrm{d} \omega$, which lie between angular frequencies $\omega$ and $\omega+\mathrm{d} \omega$, may be found (Figure 1.2). Inelastic X-ray scattering from diamond powders, of 3 to $5 \mu \mathrm{m}$ size, has been used to derive the density of phonon states more directly from experiment [27]. The results are in very close agreement with the calculated density [7, 26], the major difference being a smaller measured peak in $g(\omega)$ at the highest frequencies compared with calculation.

\subsubsection{Special values of $k$ : the Raman frequency}

Since diamond is a homopolar solid, a pure macroscopic diamond does not have any electrostatic dipole moments. The derivative of the dipole moment with respect to any deformation is also zero, and so diamond does not satisfy the necessary condition for first-order absorption of infrared radiation with the creation of one phonon in a pure bulk sample; impurities allow absorption with the creation one phonon, as would nano-scale dimensions. Pure diamond may absorb radiation with the involvement of more than one phonon. Qualitatively, one phonon can be thought to distort the lattice, creating instantaneous dipoles, to which the infrared radiation may couple, creating a second phonon. The optical absorption spectrum is then measuring combinations of phonons rather than single phonons. However, the creation of one phonon is allowed in Raman spectroscopy. 
Pure diamond has one Raman-active mode, producing a sharp peak of full width at half height, at room temperature, in high quality crystals, of $\Gamma \sim 1.2 \mathrm{~cm}^{-1}$, at $\omega_{R}=$ 1332.4(1) $\mathrm{cm}^{-1}$. Micro-Raman measurements, extrapolated to $0 \mathrm{~K}$, give a limiting line width of $1.10(3) \mathrm{cm}^{-1}$ [28]. The line width is determined, in a crystal, by lifetime broadening. A third-order perturbation approach [29], in which the Raman mode decays into two phonons, has predicted a decay time of $5.2 \mathrm{ps}$ and so $\Gamma=1.0 \mathrm{~cm}^{-1}$. Direct observation of the decay of the Raman line has been reported by pumping a pure diamond with $395 \mathrm{~nm}$ light, and observing a small (parts per million) oscillation in the reflection coefficient, the time period of the oscillation being equal to the Raman frequency [30]. The oscillations decayed at room temperature with an exponential decay time of $6.9 \mathrm{ps}$. Because the acoustic branches in diamond rise to a high value relative to the optic modes compared with other semiconductors (Section 1.3), there is a high density of acoustic modes at half the Raman energy. The Raman phonon predominantly decays into two acoustic phonons of similar energy [29], as in the 'Klemens' model [31].

The high intensity and sharpness of the Raman line has allowed detailed measurements to be made on it. With these data, the Raman line may be used to measure the temperature of a diamond, or to measure a high pressure that the diamond is subjected to. For example, the pressure in a diamond anvil cell may be measured using the exact frequency of the Raman line from a ${ }^{13} \mathrm{C}$ diamond chip that has been included in the working volume of the cell [32], or by using the Raman signal from a ${ }^{13} \mathrm{C}$ isotopically enhanced diamond anvil [33].

The dependence of a frequency of vibration on the volume $V$ of the crystal is usually expressed through the 'Grüneisen parameter' $\gamma$ of the mode:

$$
\frac{\Delta \omega}{\omega}=-\gamma \frac{\Delta V}{V} .
$$

Data have been presented by many workers [34-38] using the hydrostatic compressive stress $P$ as the controlled variable, with agreement for several data sets now up to 140 $\mathrm{GPa}$ at [15]:

$$
\frac{\Delta \omega}{\Delta P}=2.7 \text { to } 2.9 \mathrm{~cm}^{-1} / \mathrm{GPa},
$$

corresponding, via Equation (1.13), to $\gamma=-0.90$ to -0.97 . Occelli et al. [15] have taken the pressure sufficiently high that nonlinear effects are very pronounced, giving, with $P$ in GPa:

$$
\omega=1333.0+2.83 P-3.65 \times 10^{-3} P^{2} \mathrm{~cm}^{-1} .
$$

The Grüneisen parameter is effectively constant at $\gamma=-0.97(1)$ throughout the range. Values of $\gamma=-0.92$ to -0.99 have been calculated using quantum Monte Carlo calculations [16].

The full width of the Raman line at half its width is sample dependent, for example, through the variations in strain fields, but has a minimum value of $\sim 1.2 \mathrm{~cm}^{-1}$ in the limit of low temperature (essentially below room temperature). The decay at low $T$ is reported above to be into two modes of equal energy, hence $667 \mathrm{~cm}^{-1}$, and the probability of emitting those phonons increases as $\bar{n}$, see Equation (1.20). Above room temperature, the 
line width increases with temperature. Data for the line width up to $900 \mathrm{~K}$, by Liu et al. [39], agree with the third-order perturbation calculation of Debernardi et al. [29] and are less temperature dependent than earlier data [40, 41].

The Raman frequency is triply degenerate in diamond, and so is split into a nondegenerate state and a doubly degenerate state when the symmetry is lowered by compression along the $\langle 111\rangle$ and $\langle 001\rangle$ axes. The splittings [42,43] are $\Delta \omega=2.2 \pm 0.2 \mathrm{~cm}^{-1} \mathrm{GPa}^{-1}$ for $\langle 111\rangle, \Delta \omega_{001}=0.73 \pm 0.1 \mathrm{~cm}^{-1} \mathrm{GPa}^{-1}$ for $\langle 001\rangle$, comparable with the values in Equation (1.23). Note that for the same applied stress, the fractional changes $\Delta \omega / \omega$ are typically two orders of magnitude smaller for vibrations than for electronic states. Nevertheless, the splitting can be used to probe the stress inhomogeneities in diamond anvil cells [44].

A uniaxial stress produces a perturbation with even parity, while an electric field has odd parity: reducing the symmetry by an electric field allows the Raman frequency to be observed in optical absorption, the intensity of absorption increasing as the square of the applied field, and with a spectrometer-corrected line width of $\sim 1.5 \mathrm{~cm}^{-1}$ [45].

The small width of the Raman line allows it to be studied as a function of temperature. At high temperature, the temperature of the sample may be measured using the ratio of the probabilities of emitting (Stokes) or absorbing (anti-Stokes) a phonon in the Raman processes. The intensity $I$ of the Raman line depends on the fourth power of its photon energy, and so the ratio is given by Equation (1.21) modified by a term containing the laser excitation frequency $\omega_{L}$ :

$$
\frac{I_{e}}{I_{a}}=\left(\frac{\omega_{L}-\omega_{R}}{\omega_{L}+\omega_{R}}\right)^{4} \exp \left(\frac{\hbar \omega_{R}}{k_{B} T}\right) .
$$

Data by Zouboulis and Grimsditch [40] for the Raman frequency from room temperature to $1850 \mathrm{~K}$ are in close agreement with data by Herschen and Capelli [41], who fitted their measurements to the simple expression:

$$
\omega_{R}(T)=\omega_{R}(0)-0.00777 T-1.075 \times 10^{-5} T^{2}, 300<T<1850 \mathrm{~K}
$$

where $\omega_{R}(0)=1334.5 \mathrm{~cm}^{-1}$.

The origin of the shift is partly caused by the expansion of the lattice, which, above room temperature, may be represented by a power series (Equation 1.6), as in Equation (1.26). However, evaluation using the Grüneisen parameter, after Equation (1.23), shows that the expansion accounts for only about one third of the observed effect, the remainder being caused by the anharmonicity of the vibrations.

Raman scattering is frequently used in assessing the crystallinity of chemical-vapour deposited diamond, by considering the ratio of the Raman lines from diamond and from noncrystalline (or nondiamondiferous) material [46]. The efficiency of exciting the two Raman features varies with the excitation wavelength, with UV favouring diamond and near infrared favouring nondiamond [47].

\subsubsection{Special values of $k$ : other modes}

When, at low temperature, an electron is excited to the conduction band, it will de-excite rapidly (in picoseconds) to the minimum in the conduction band, located at a wave vector 
$\mathbf{k}=0.76 \pm 0.002$ of the zone-boundary value along the $\langle 001\rangle$ axes. The hole will similarly de-excite, to its minimum energy state in the valence band at $\mathbf{k}=0$. Recombination of the electron and hole in the pure diamond requires the wave vector to be conserved by emitting a phonon with $\mathbf{k}_{001}=0.76 \pm 0.002$. Selection rules result in three of the phonons at that wave vector being identifiable: the transverse acoustic, transverse optic and longitudinal optic. The energy $(h v)$ of the photon emitted by the recombination of the electron and hole is then reduced from the free-exciton band-gap energy $E_{b}$ by the energy $\hbar \omega$ of each phonon: $h v=E_{b}-\hbar \omega$. However, because the recombination without involving a phonon is forbidden, there is uncertainty in the value of $\hbar \omega$. It may be measured from the onset of the equivalent absorption process, at $h v=E_{b}+\hbar \omega[48]$ :

$$
\hbar \omega_{\mathrm{TA}}=700 \pm 15, \hbar \omega_{\mathrm{TO}}=1137 \pm 15, \hbar \omega_{\mathrm{LO}}=1315 \pm 10, \mathrm{~cm}^{-1} .
$$

One problem with using the recombination of the free exciton is that the exciton has kinetic energy as a result of its thermal energy, leading to a line width of $1.8 k_{\mathrm{B}} T$ [49]. More precise values may be determined by using the excitons that are weakly bound to the shallow boron acceptor, if we assume that the localisation does not affect the energy of the phonons involved. The localisation does, however, induce a zero-phonon component, providing a useful origin in the energy scale from which to measure the phonon energies. By this method, the energies in natural diamond are [50]:

$$
\hbar \omega_{\mathrm{TO}}=1139 \pm 5, \hbar \omega_{\mathrm{LO}}=1315 \pm 5, \mathrm{~cm}^{-1} .
$$

The width of the phonon-assisted transitions in the bound-exciton luminescence allows the dispersion in $k$-space of those modes to be detected [51].

\subsubsection{Two- and three-phonon processes}

The two-phonon Raman scattering spectrum of diamond is well known [11, 20] and has been fitted closely, using five parameters to describe the second-order polarisability [22]. By definition, the multi-phonon processes involve combinations of (usually different) phonons. Identifications of the individual phonons involved have been attempted by associating peaks in the two-phonon absorption spectrum with peaks in the density of phonon states, either from an experimental matching or, more formally, from the calculated spectra. For example, Tables III of Ref. [23] and II of Ref. [22] contain extensive identifications. To avoid using combinations of modes, Klein et al. [52] have used the one-phonon, defect-induced, infrared absorption in imperfectly grown chemical-vapour deposited diamonds to pick out critical points in the one-phonon density of states.

Measurements [53] on the two-phonon Raman spectrum under hydrostatic stress (of $<2.4 \mathrm{GPa}$ ) have given shift rates for the combined modes of 5.6 to $8.6 \pm 0.6 \mathrm{~cm}^{-1} \mathrm{GPa}^{-1}$; we note that the same work resulted in a shift rate for the Raman line of about $20 \%$ larger than the values of Equation (1.24), and so a $20 \%$ reduction in the shift rates should probably be applied. The data are in overall agreement with the results of ab initio calculations of the Grüneisen parameters (Figure 1.3). These calculations suggest that the 


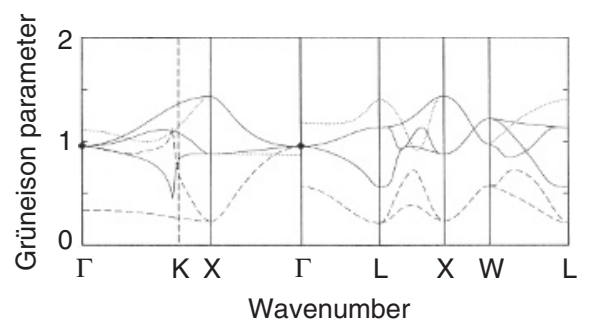

Figure 1.3 Grüneisen parameters for diamond at room temperature, as calculated by Pavone et al. [7]. The dashed lines are transverse acoustic modes, dotted lines are longitudinal acoustic modes, and solid lines are optic modes. The experimental data for the Raman mode are shown by diamonds

Grüneisen parameters $\gamma$ for transverse acoustic, longitudinal acoustic and optic modes lie in the approximate ranges:

$$
0.2<\gamma_{\mathrm{TA}}<1.1,1.0<\gamma_{\mathrm{LA}}<1.4,0.5<\gamma_{\mathrm{TA}}<1.4
$$

We noted that photons may be absorbed by a perfect diamond with the creation of two or more phonons. The shape of the absorption spectrum is well known experimentally (see, for example, Ref. [54]), but has not yet been closely simulated by theory. We would expect that the combinations of phonons must conserve wave vector, and that peaks in the two-phonon spectrum would follow the peaks in the two-phonon density of states. However, the band shape is strongly dependent on the transition probability for creating each pair of phonons. The absorption features move to lower energy as the temperature increases, as a result of the anharmonicity of the vibrations [55] and the lattice expansion. Typically lattice expansion accounts for about half of the total shift, and a simple parameterisation for the total change in energy $E$ is of the form:

$$
\Delta E=C /\left[\exp \left(\hbar \omega / k_{\mathrm{B}} T\right)-1\right]
$$

where $\hbar \omega$ is an effective, (lowest energy), phonon involved in the process. All the distinctive features of the two-phonon spectrum move to lower energy at approximately the same fractional rates:

$$
\Delta E / E \sim-0.027 /\left[\exp \left(860 \mathrm{~cm}^{-1} / k_{\mathrm{B}} T\right)-1\right] .
$$

For the well-defined features produced by the creation of two phonons, the absorption coefficient $A(v, T)$ increases with temperature $T$, as expected from Equation (1.21):

$$
A(v, T)=a(v, 0)\left[1+n\left(E_{1}, T\right)\right]\left[1+n\left(E_{2}, T\right)\right],
$$

where $E_{1}$ and $E_{2}$ are the phonon energies, and of course $h v=E_{1}+E_{2}$.

An especially important two-phonon absorption process is that at $10.6 \mu \mathrm{m}, 943 \mathrm{~cm}^{-1}$, the frequency of a $\mathrm{CO}_{2}$ laser. The absorption in pure diamond is $\sim 0.03 \mathrm{~cm}^{-1}$ at $300 \mathrm{~K}$, but increases very rapidly, being a factor of 9 larger at $800 \mathrm{~K}$ [54]. Since wave vector and energy must be conserved, the two phonons involved must have, from the dispersion 
curves, energy quanta of $E_{1}=400 \pm 30 \mathrm{~cm}^{-1}$ and $E_{2}=540 \mp 30 \mathrm{~cm}^{-1}$. The temperature dependence of the absorption with these two phonons being created is then given by Equation (1.32) and amounts to a factor of 2.8 increase between 300 and $800 \mathrm{~K}$, and not the factor of 9 observed. The difference is caused partly by the two-phonon process at $943 \mathrm{~cm}^{-1}$ involving the destruction of one phonon and the creation of a second. From the dispersion curves, wave vector and energy may be conserved by destroying one low-energy acoustic mode of $E_{1}=335 \pm 35 \mathrm{~cm}^{-1}$ and creating an optic mode of $E_{2}=1272 \pm 35 \mathrm{~cm}^{-1}$. The absorption coefficient of this process then increases with temperature as (see Equation 1.22):

$$
A(\nu, T)=a(v, 0) n\left(E_{1}, T\right)\left[1+n\left(E_{2}, T\right)\right],
$$

and the low value of $E_{1}$ produces considerable temperature dependence. A further process contributing to the rapid rise in absorption with $T$ at $943 \mathrm{~cm}^{-1}$ is that the phonon frequencies drift downwards as $T$ increases (Equation 1.30), moving the stronger absorption that occurs at higher energy down to $943 \mathrm{~cm}^{-1}$. Finally, with increasing $T$, the low-energy, three-phonon, processes (discussed next) become significant. The very strong temperature dependence can therefore be understood explicitly in terms of known components.

For the three-phonon processes, the allowed combinations of phonons are apparently sufficiently large that one may simply use the density $g_{3}(\omega)$ of three-phonon states, derived by twice convoluting the one-phonon density with itself. The absorption coefficient $A(v)$ at photon frequency $v$ is then found to be proportional to the combined density $g_{3}$ multiplied by the fourth power of $v$. With increasing temperature, the probability of emitting each phonon increases as in Equation (1.21), but in practice it is sufficient to use a frequency $v / 3$ for each of the phonons. The absorption then depends on the photon frequency and the temperature $T$ as [54]:

$$
A(v) \propto v^{4} g_{3}(v)[1+n(v / 3)]^{3} .
$$

\subsubsection{Isotope effects}

Multi-phonon Raman and infrared absorption measurements on isotopically modified diamonds produce the same band shapes but with the frequency scale modified essentially in accordance with the 'virtual crystal approximation', in which the mean mass of the lattice atoms is used [23]. Thus, for a mixture of $x$ parts of ${ }^{13} \mathrm{C}$ and $(1-x)$ parts of ${ }^{12} \mathrm{C}$, the mean mass is $12+x$ and the frequencies in this approximation are:

$$
\omega(x)=\omega_{12} \sqrt{\frac{12}{12+x}} .
$$

For $\omega_{12}=1332.8 \mathrm{~cm}^{-1}, \omega(x)=1332.8-55.5 x+3.5 x^{2}$. Precise measurements may be made on the one-phonon Raman line [23, 56-58]. The peak frequency is fitted with:

$$
\omega(x)=1332.82-34.77 x-16.98 x^{2} \mathrm{~cm}^{-1} .
$$

The shift is made up of the dominant virtual crystal term, plus the static lattice contraction affecting the frequency of the mode (the Grüneisen effect). Additionally, isotope disorder 
increases the scattering of phonons (Section 3.7 below). Ruf et al. [59] give a simple description of the effect by representing it in terms of a two-level model. One level, say the Raman phonon, interacts with other modes, represented by the second level, which is located at about the mean of the density of phonon states. Since the Raman frequency is greater than that mean, the interaction produced by the isotope disorder will force the Raman frequency higher. Measurement and theory [59] show that the disorder contributes a shift to the Raman line of between +4 and $+7 \mathrm{~cm}^{-1}$ at $x \sim 0.5$. The disorder effects occur through the minority isotope species. For example, at high $x$ the scattering atoms are ${ }^{12} \mathrm{C}$, which raise the frequency of the active modes relative to the values in the pure ${ }^{13} \mathrm{C}$ crystal. The scattering modes are close to the Raman energy and produce a large positive shift. In contrast, at small $x$, the 'impurity' atoms are ${ }^{13} \mathrm{C}$, which lower the frequency of the active modes away from the Raman energy. The disorder shift is asymmetric, maximising near $x=0.6$ in this case [57]. From the measured disorder broadening, at $x=0$ the line width $\Gamma$ is expected to change at the rate $\mathrm{d} \Gamma / \mathrm{d} x=2 \mathrm{meV}$, so that in a natural diamond the isotope disorder contributes about $0.16 \mathrm{~cm}^{-1}$, or over $10 \%$, to the line width.

The phonons that play an important role in the band-gap optical transitions at 0.76 of the $\langle 001\rangle$ zone boundary are shifted, within experimental error, in accordance with the virtual crystal approximation when a complete change of isotope is made $(x=0$ to $x=1)[50,59]$. Since the transverse optic mode lies below the mean in the density of states, the disorder interaction will now move that mode to lower energy [59], giving a disorder contribution at $x=0.5$ of about $-4 \mathrm{~cm}^{-1}$.

\subsubsection{Specific heat}

Knowing the density of phonon states, $g(\omega)$ (Figure 1.2), and the probability of occupying them (Equation 1.19), the energy stored in the vibrations is given by:

$$
E(T)=\int_{0}^{\omega_{\max }} \mathrm{d} \omega g(\omega) n(\omega),
$$

and the specific heat is $C \propto \mathrm{d} E / \mathrm{d} T$. If $g(\omega)$ is known, the calculation is simple. For example, a very good fit to the experimental data results from using $g(\omega)$ obtained by inelastic X-rays (Figure 6 of Ref. [27]).

At low $T$, only the low frequency acoustic modes are thermally populated, with $\omega \propto k$. Consequently, the density of states $g(\omega) \propto \omega^{2}$. This form leads to the convention of using the Debye approximation, in which the density $g(\omega) \propto \omega^{2}$ is assumed to apply to all the modes. The expression for the specific heat may then be fitted to the experimental data at any temperature by choosing the appropriate value of $\omega_{\max }$ in the integral. The value of $\omega_{\max }$ is expressed conventionally in terms of the Debye temperature, $\Theta_{D}=\hbar \omega_{\max } / k_{\mathrm{B}}$. The Debye temperature defined from specific heat measurements is $\Theta_{D}=1865 \pm 10 \mathrm{~K}$ in the range 300 to $800 \mathrm{~K}$, corresponding to an increase in the specific heat $C$ from $6.19 \mathrm{~J} \mathrm{~g}$-atom $^{-1} \mathrm{~K}^{-1}$ at $300 \mathrm{~K}$ to $19.29 \mathrm{~J} \mathrm{~g}$-atom $^{-1} \mathrm{~K}^{-1}$ at $800 \mathrm{~K} \mathrm{[60],} \mathrm{that} \mathrm{is} \mathrm{from} 0.5$ $\mathrm{J} \mathrm{g}^{-1} \mathrm{~K}^{-1}$ at room temperature to $1.6 \mathrm{~J} \mathrm{~g} \mathrm{~g}^{-1} \mathrm{~K}^{-1}$ at $500{ }^{\circ} \mathrm{C}$. To approach $90 \%$ of the classical value of $C=3 R$, temperatures in excess of $1300 \mathrm{~K}$ would be required. Below $300 \mathrm{~K}, C$ decreases rapidly as fewer vibrational modes may be thermally populated [61, 62]. The rapid decrease away from the classical value of $C_{v}=3 R$, known since 1875 
[63], is historically important as being the data that led Einstein to develop his quantum theory of specific heats [64].

The specific heat of chemical-vapour deposited diamond will depend on the quality of the films. However, the colour of diamond may be determined by very low concentrations of impurities, of the level of parts per million, which are irrelevant to bulk specific heat measurements. The specific heat of 'black' CVD diamond has been reported to be within $1 \%$ (the experimental uncertainty) of that of bulk diamond [65].

There is a significant dependence of the specific heat of diamond on its isotope content. When the isotopic content is changed by increasing the fraction of ${ }^{13} \mathrm{C}$, the predominant effect is that the frequencies of vibration are reduced in accordance with the virtual crystal approximation, and so at low temperature, where the frequency of the modes is most important, the specific heat is increased. Predictions by Sanati et al. [66] have been closely verified by measurements of the specific heat over the range 75 to $300 \mathrm{~K}$ [67]. At $170 \mathrm{~K}$, the specific heat of ${ }^{13} \mathrm{C}$ is about one-sixth larger than for ${ }^{12} \mathrm{C}$ [67].

\subsubsection{Thermal conductivity}

The standard approach to the thermal conductivity $\kappa$ of a solid is to use the analogy to conductivity in a gas:

$$
\kappa=\frac{1}{3} C v^{2} \tau
$$

where $C$ is the specific heat, $v$ is the speed of the heat carriers (phonons in diamond), and $\tau$ is the mean time between scattering events for the carriers. In diamond, there will be different contributions from the different frequencies of the phonons, requiring a sum over all the densities of state [68].

The specific heat increases from zero at $0 \mathrm{~K}$, becoming a constant at high temperature (Section 1.3.6). With increasing $T$, there is an increase in the number of phonons available to transport the heat, and those phonons have increasing frequencies as $T$ increases. However, with the increased populations of phonons, the probability of interacting with another phonon increases, and the probability of decaying into other phonons also increases (Equation 1.21). These intrinsic processes result in the phonon population being $\sim \hbar \omega / k_{\mathrm{B}} T$ at high $T$, and so $\tau \propto T^{-1}$. At sufficiently high $T$, approximately above room temperature, this process will dominate in any diamond, and the thermal conductivity will be independent of the sample. Below about $100 \mathrm{~K}$, only relatively low frequency (long wavelength) phonons are thermally excited. At this limit, the dimensions of the sample determine the scattering time; for example, polycrystalline diamond films could, therefore, have an anisotropic thermal conductivity. Finally, at intermediate temperatures, $T \sim 50$ to $300 \mathrm{~K}$, the scattering time $\tau$ is determined by the impurities or other crystalline imperfections in the diamond, including isotope disorder. Although its peak thermal conductivity is at $70 \mathrm{~K}$, the thermal conductivity of diamond at room temperature is still higher than for any other solid, and over four times that of pure copper.

These processes imply that the value of $\kappa$ is, below $300 \mathrm{~K}$, highly dependent on the specific sample used. There are now many techniques in use for determining the thermal conductivity, as reviewed by Reichling and Hartmann [69]. In general terms, high quality, 'pure' (type IIa) natural diamonds have their maximum thermal conductivity of 
$\sim 15000 \mathrm{~W} \mathrm{~m} \mathrm{~m}^{-1} \mathrm{~K}^{-1}$ at $\sim 70 \mathrm{~K}[70-72]$. Above $100 \mathrm{~K}$, in high quality natural samples, $\kappa$ decreases approximately inversely with $T$, to $\sim 600 \mathrm{~W} \mathrm{~m}^{-1} \mathrm{~K}^{-1}$ at $\sim 1000 \mathrm{~K}$ [70-72]. Diamonds are likely to contain atomic-sized defects and impurities, which act as scattering centres. They are most important in reducing the thermal conductivity in the central range, where neither the crystal size nor the phonon-phonon scattering is dominant. For example, reductions in the maximum value of $\kappa$ by an order of magnitude are easily obtained in natural 'type Ia' diamonds with concentrations of nitrogen of a few parts per thousand. Similarly, radiation damage introduces additional thermal resistance in proportion to the concentration of vacancies [73].

Isotope disorder creates significant scattering of the phonons, reducing the thermal conductivity. Again, at the limits of low and high temperatures, $\kappa$ is expected to be independent of the isotope content. However, an isotopic 'impurity', such as a ${ }^{13} \mathrm{C}$ atom in a predominantly ${ }^{12} \mathrm{C}$ diamond, is expected to increase the scattering rate $\tau^{-1}$ in proportion to the concentration of the isotopic impurity, and as the fourth power of the phonon frequency. The thermal conductivity is substantially reduced by the disorder near its maximum values (typically between 30 and $200 \mathrm{~K}$ ). Isotopically enhanced diamond, with only $0.1 \%{ }^{13} \mathrm{C}$ has a peak value of $\kappa$ some three times larger than diamond of natural isotope abundance [74], and the peak is shifted slightly, but possibly usefully, to higher $T$, to about $90 \mathrm{~K}$. Isotopically pure diamond $\left({ }^{13} \mathrm{C}\right.$ less than $\left.0.001 \%\right)$ would probably have a thermal conductivity of more than an order of magnitude greater than a diamond of natural isotope abundance.

In polycrystalline films, which have nucleated at many points on the surface of the substrate, the thermal conductivity may be highly anisotropic. A typical cross-section of the film will show many small crystallites at the nucleation surface, of which only a small proportion will grow into elongated crystals to the surface of the film. In such a sample, we could expect that the thermal conductivity measured through the thickness of the film, along a single crystal, may be significantly higher than in the lateral direction, where the heat flow has to jump across grain boundaries [75]. In this case, the resistance $\kappa_{F}^{-1}$ to heat flow in the plane of the film is then made up of the resistance $\kappa^{-1}$ inside the (pure) diamond grain, plus the resistance from the grain interfaces:

$$
\kappa_{F}^{-1}=\kappa^{-1}+\frac{R_{\mathrm{th}}}{a} .
$$

Here, $R_{\mathrm{th}}$ is the resistance of one interface, and $a$ is the mean grain size. For example, in samples investigated by Hartmann et al. [76], the interfacial resistance was $2 \times 10^{-9} \mathrm{~m}^{2} \mathrm{~K}$ $\mathrm{W}^{-1}$, and so became increasingly important at grain sizes of $a<20 \mu \mathrm{m}$. For this value of $R_{\text {th }}$, the room temperature value of $\kappa_{F}^{-1}$ decreases by a factor of two between 9 and $2.5 \mu \mathrm{m}$. However, if the first 30 to $40 \mu \mathrm{m}$ of the CVD crystal growth is removed from the samples, and good quality material is used, the thermal conductivity in the plane of the CVD film and perpendicular to that plane have been found to be equal, within a measuring accuracy of $8 \%$ [77]. Room-temperature values of $\kappa$ of up to $2220 \mathrm{~W} \mathrm{~m} \mathrm{~m}^{-1} \mathrm{~K}^{-1}$ were reported [77], which very closely approach the value for good natural crystals $\left(\sim 2265 \mathrm{~W} \mathrm{~m} \mathrm{~m}^{-1} \mathrm{~K}^{-1}\right)$. Twitchen et al. [77] observed an excellent correlation in their samples between the thermal conductivity and the concentration of the carbon-hydrogen complexes identified by the absorption in the vibrational transitions at 2760 to $3030 \mathrm{~cm}^{-1}$; these complexes appeared to be the dominant extrinsic source of thermal resistance. In earlier work, in some batches 
of polycrystalline films contaminated with nondiamond carbon, the ratio of the Raman signals from the diamond and nondiamond components was found to be a good indicator of the lateral thermal conductivity [78]: an example of how the limitations of materials decrease as the technology improves. As for single crystal diamond, isotope disorder in films can have a measurable effect on the thermal conductivity, and Belay et al. [79] have reported a lateral thermal conductivity three times lower in a $50 \%{ }^{13} \mathrm{C}$ sample compared with a sample of natural isotopes.

At the nanoscale, the surfaces become extremely significant, both in terms of surface contaminants and the intrinsic ability of carbon to rehybridise, and so the method of preparation is a key factor. The sparse data available to date show that the surfaces present significant barriers to thermal diffusion, resulting in very low thermal conductivity $(\sim 0.1$ $\mathrm{W} \mathrm{cm}^{-1} \mathrm{~K}^{-1}$ at room temperature [28]).

\subsection{ELECTRONIC ENERGY STATES}

\subsubsection{Dispersion curves}

The valence bands in diamond have their maxima at $\mathbf{k}=0$, and the minimum in the first conduction band at $\mathbf{k}=0.76$ of the zone boundary in the $\langle 001\rangle$ directions [48]. The energy gap at low temperature is $5.49 \mathrm{eV}$, much larger than the other Group IV semiconductors, while the spin-orbit splitting at the maximum of the valence band is particularly small at $\sim 12 \mathrm{meV}$ [80].

Experimental data have been derived mainly from optical measurements of absorption, luminescence, reflectance and ellipsometry. Given the indirect nature of the lowest band gap, optical absorption must involve the creation or (at high enough temperature) the destruction of a wave-vector-conserving phonon (Section 1.3.3). The absorption coefficient of pure diamond increases rapidly with photon energy, as discussed by Clark et al. [81]. Diamond is effectively opaque at photon energies just above the indirect band gap, but data on the band structure were obtained many years ago by using reflection spectra up to $35 \mathrm{eV}[82,83]$. From the reflection spectra, the real (reflectance) and imaginary (absorbance) parts of the dielectric function can be obtained. Sharp features in the function derive primarily from the joint (valence and conduction band) density of states, and so relate to either the extreme of a band or to energy-wave vector plots of the valence and conduction bands running parallel to each other in k-space. Distinguishing between them relies on band structure analyses (Figure 1.4). The lowest direct gap is located at $7.2 \mathrm{eV}$ above the valence band maximum. Good fits to the dielectric function between 5 and 25 $\mathrm{eV}$ are given by Papadopoulos and Anastassakis [84].

The width of the valence band has been measured using photoelectron spectroscopy by several groups. The width of the valence band, as determined by photoexcitation [85], is $23.0 \pm 0.2 \mathrm{eV}$, greater than the width of $21.5 \pm 0.2 \mathrm{eV}$ predicted by local-density functional theory [86, 87], which does not include the many-particle contributions to the excitation. The exact shapes of the bands near the valence band maximum and first conduction band minimum have been predicted [88].

Formally, the effective mass of an electron or hole is given by the inverse curvature of the dispersion plots, $m_{i j}^{\star}=\hbar^{2} /\left(d^{2} E / d k_{i} d k_{j}\right)$. For the electron at the minimum of the conduction band, two masses are required, corresponding to motion longitudinal $(\mathbf{k}||\langle 001\rangle$ 


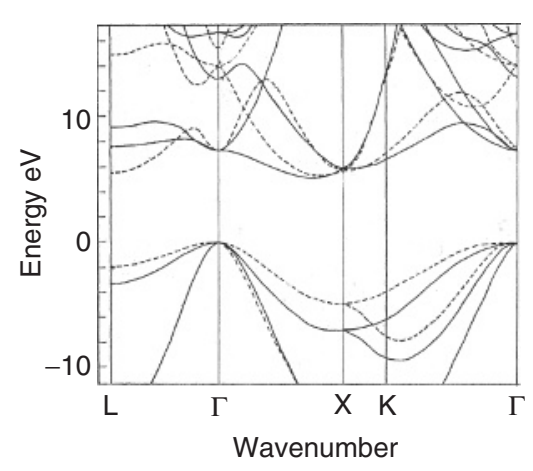

Figure 1.4 Electronic energy bands in pure diamond, for wave vectors $\mathbf{k}$ parallel to the major crystallographic axes. Point $\Gamma$ has $\mathbf{k}=0$, and $X, L$ and $\mathrm{K}$ are respectively at the $\langle 001\rangle,\langle 111\rangle$ and $\langle 110\rangle$ zone boundaries. The lines are calulated by L.R. Saravia and D. Brust, Phys. Rev . 170, 683 (1968)

for the $\langle 001\rangle$ minimum) or to transverse motion. Experimental masses for the electron have been calculated from the electron drift velocity by Nava et al. [89], yielding:

$$
m_{t, e}^{\star}=0.36 m_{0}, m_{l, e}^{\star}=1.4 m_{0}
$$

in terms of the vacuum mass $m_{0}$ of the electron. One application of the masses is their use in predicting the higher energy levels of electrons bound to donors, using effective mass theory. A fit to experimental data for the energy levels of the phosphorus donor yields [90] $m_{t, e}^{\star}=0.31 m_{0}$, and $m_{l, e}^{\star}=1.8 m_{0}$ in reasonable agreement. Further, a detailed theoretical investigation of the band extrema [88] results in $m_{t, e}^{\star}=0.34 m_{0}$, and $m_{l, e}^{\star}=1.5 m_{0}$, in close agreement with Equation (1.40).

The valence band maximum is complicated by being composed of $\mathrm{p}$ atomic orbitals, giving, in atomic language, spin-orbit split states of $j=3 / 2$ (lower energy hole states) and $j=1 / 2$. The spin-orbit splitting is small, so that both the $j=3 / 2$ and the $j=$ $1 / 2$ states need to be considered in any physical process, such as the formation of the bound states of the hole at the boron acceptor. Further, the $j=3 / 2$ states split into $m_{j}= \pm 3 / 2, m_{j}= \pm 1 / 2$ pairs when the symmetry is reduced by having $k \neq 0$. To obtain different values of the energy at the same value of $\mathbf{k}$ requires different values of the curvature of the valence band for the $j=3 / 2$ states, and hence two masses, a heavy hole (smaller hole energy) and a light hole. The tensor property of the effective mass is a further complication. Values derived theoretically for motion along the $\langle 001\rangle,\langle 111\rangle$ and $\langle 110\rangle$ directions are, for the heavy hole [88]:

$$
m_{001}^{\star}=0.43 m_{0}, m_{111}^{\star}=0.78 m_{0}, m_{110}^{\star}=0.69 m_{0},
$$

and for the light hole

$$
m_{001}^{\star}=0.37 m_{0}, m_{111}^{\star}=0.26 m_{0}, m_{110}^{\star}=0.28 m_{0} .
$$

The split-off spin-orbit state has a mass $0.4 m_{0}$ [88].

The value of the spin-orbit interaction has been subject to some controversy. Considering only the maximum of the valence band, the atomic properties to consider are the 
angular momentum of the $\mathrm{p}$ orbitals, and the spin of the electron. They couple to give the $j=3 / 2$ and $j=1 / 2$ states, split at $\mathbf{k}=0$ by the spin-orbit interaction $\lambda$. In an experiment, further interactions may be involved. For example, when a free exciton is created (Section 1.4.3 below), the hole at the valence band maximum interacts with an electron, which has at least angular momentum in its spin. Exchange coupling between the spins of the hole and the electron, of magnitude $\Delta$, would, in the absence of spin-orbit coupling, create a spin singlet, $S=0$, and a spin triplet, $S=1$. These two interactions need to be considered together. A re-analysis by Cardona et al. [80] of data by Sauer et al. [91] suggests that the spin-orbit interaction is:

$$
\lambda=11.7 \mathrm{meV}
$$

in close agreement with theoretical calculations [88].

The lowest (indirect) energy gap moves to higher energy under hydrostatic pressure $P$ at the rate, at room temperature and from $P=0$ to $P=2.3 \mathrm{GPa}$, of [92]:

$$
\mathrm{d} E / \mathrm{d} P=6.0 \mathrm{meV} / \mathrm{GPa}
$$

an order of magnitude larger than the shifts for the phonons (Equation 1.23).

When an electron has been excited from the maximum in the valence band to an energy state with more than twice the direct energy gap, its lifetime in that state has been estimated (from lifetime broadening) to be a few times $10^{-16} \mathrm{~s}$ [93]. The high energy state allows de-excitation of the electron through electron-electron interactions.

In nano-diamond, the surface properties become highly significant, in addition to the dimensions of the sample. For example, the band gap is predicted to decrease in diamond nanowires relative to bulk diamond, the extent depending on these properties [94].

\subsubsection{Temperature dependence and isotope dependence of the lowest energy gap}

With increasing temperature $T$, the lowest (indirect) energy gap at $5.49 \mathrm{eV}$ moves monotonically to lower energy [81]. A shift of $-110 \mathrm{meV}$ is reported by $675 \mathrm{~K}$. The effect is partly made up of the effect of lattice expansion (Section 1.2.2). A volume change of $\Delta V$ produces a shift:

$$
\Delta E_{\exp }=-a\left(c_{11}+2 c_{12}\right) \Delta V / V
$$

where the elastic constants are known (Section 1.2.4). There is uncertainty in $a$, the change in energy of the energy gap per unit hydrostatic pressure, with $a \sim 5$ to $6.4 \mathrm{meV} / \mathrm{GPa}$ $[50,95]$. However, the lattice expansion term accounts for less than $10 \%$ of the total observed shift. The majority of the shift comes from electron-phonon interactions. These have been discussed in detail by Zollner et al. [96], showing that total electron-phonon coupling in the valence band is about double that in the conduction band, and the coupling is predominantly in the optic modes. Their calculation reproduces the measured data. The electron-phonon coupling may be parameterised in terms of the density $g(\omega)$ of phonon states (Section 1.3.1), and the Bose-Einstein population term $n(\omega)$ (Equation 1.20). A 
simple empirical expression shows that the coupling term is proportional to the frequency $\omega$ of the phonon, giving:

$$
\Delta E_{\mathrm{e}-\mathrm{p}}=c \int \mathrm{d} \omega \omega g(\omega) n(\omega),
$$

where the integral is over all the frequencies. This expression allows the energy-gap renormalisation energy to be found $(\sim 370 \mathrm{meV})$, which is required for discussion of the isotope effects. However, for practical purposes, the expression of O'Donnell and Chen [97] provides a simpler fit: the shift between 100 and $700 \mathrm{~K}$ is given by $-S \hbar \omega[\operatorname{coth}(\hbar \omega / k T)-1]$ with $S=2.31$ and $\hbar \omega=94 \mathrm{meV}$. Based on extraordinarily high precision measurements in single-isotope silicon, Cardona et al. [67] have proposed a universal law to the effect that the energy gap will change as $T^{4}$ at very low (liquid helium) temperatures.

Closely related to the temperature dependence is the isotope dependence. The total shift of the lowest indirect energy gap from natural to ${ }^{13} \mathrm{C}$ diamond is measured, at low $T$ to be [50]:

$$
E_{13}-E_{12}=13.6 \pm 0.2 \mathrm{meV}
$$

Measurements by Ruf et al. [98] of luminescence from excitons weakly bound to boron acceptors show that their energies, which closely follow the lowest indirect energy gap, change linearly with the ${ }^{13} \mathrm{C}$ isotopic fraction $x$ at a shift rate of $\mathrm{d} E / \mathrm{d} x=+14.6 \pm 0.5$ meV.

The shift is again partly caused by the contraction of the lattice that occurs when the isotope is changed (Section 1.2.3), but this is a small contribution of $3 \pm 1.3 \mathrm{meV}$ to the total shift [50]. Most of the shift can be identified as a result of changing the vibrational frequencies in Equation (1.46), and by using the value of $c$ from the temperature dependence, a contribution of $+13.5 \pm 2 \mathrm{meV}$ was estimated to come from the electron-phonon term. A very similar value of $+18.4 \mathrm{meV}$ was predicted ab inito by Zollner et al. [96].

The temperature dependence of the first direct energy gap is similar to that of the lowest indirect gap [99].

\subsubsection{Exciton states and electron-hole condensates}

When one electron is excited to the conduction band, creating one electron at the conduction band minimum and one hole at the valence band maximum, separated in space by a distance $r$, there is a coulombic attraction between them of $V=-e^{2} / 4 \pi \varepsilon r$ for the two particles. At low temperature, a pseudo-hydrogenic system can be created with the electron and hole orbiting each other in a 'exciton' state, with a binding energy $E_{\mathrm{ex}}=e^{2} / 8 \pi \varepsilon a_{\mathrm{ex}}$. The binding energy of the exciton is measured as $80 \mathrm{meV}$ and, together with the static dielectric constant $\varepsilon_{\infty}=5.70$ from experiment [100], yields an effective radius for the exciton of:

$$
a_{\mathrm{ex}}=1.58 \mathrm{~nm} \text {. }
$$

The lifetime of the exciton has been measured as $2.3 \mu$ s [101]. The recombination of the electron and hole must involve a wave-vector conserving phonon (Equation 1.27), 
and so involves three particles plus the emitted photon (see Section 1.3.3). In silicon, free excitons may survive for tens of microseconds. In diamond, the exciton's lifetime is long enough for diffusion to take place, even at liquid helium temperature, from its random creation point to be trapped, for example, at a boron atom, when boron is present at concentrations of over $\sim 10^{17} \mathrm{~cm}^{-3}$. Since the exciton's lifetime is determined partly by the rate of capture at impurities, any experimental value should be regarded as a lower limit.

With increasing excitation power, many electrons and holes may be created before the first pair recombines. As long as sufficent power is placed in a pure diamond without appreciable heating of the sample ( $T$ less than about $165 \mathrm{~K}$ [102]), the attraction of the oppositely charged particles lowers their energy sufficiently for an 'electron-hole condensate' to be created within 'several tens of picoseconds' [102], analogous to a liquid being formed in preference to a gas (the free excitons). Luminescence from the electron-hole condensate still involves transitions across the indirect energy gap, and so involves the phonons of Equation (1.27). The electron-hole attraction lowers the available electronic energy relative to the $5.49 \mathrm{eV}$ energy gap by an amount that depends on the concentration of the electrons and holes, and hence on the excitation power. Luminescence, which grows rapidly with excitation power, is then observed in the range 5.1 to $5.25 \mathrm{eV}$, involving the transverse-optic phonon [103]. The concentration of electrons and holes in the condensate depends on the excitation and temperature, but is of the order of $10^{20} \mathrm{~cm}^{-3}[102,103]$. The decay time of the condensate, measured as $\sim 1$ ps at $15 \mathrm{~K}$, is significantly shorter than the lifetime $(2.3 \mu$ s quoted above) of the free exciton, and is consistent with quenching by the Auger process [102]. In ${ }^{13} \mathrm{C}$, luminescence from the electron-hole condensate is shifted by about $14 \mathrm{meV}$ relative to ${ }^{12} \mathrm{C}$, as expected from Equation (1.47) [103]. A thorough account of the electron-hole condensate is given by Sauer et al. [104].

Finally, we note the electronic states of an exciton, whether free or weakly bound to boron or phosphorus, sample a volume of the crystal given by $V=4 \pi a_{\mathrm{ex}}^{3} / 3$, where $a_{\mathrm{ex}}$ is known (Equation 1.46). Random isotopes inevitably broaden those states, at a rate determined by the isotope dependence of the energy gap, $\mathrm{d} E / \mathrm{d} x=13.6 \mathrm{meV}$ (Equation 1.47). The full width at half height of an optical transition in natural diamond, $x=0.01$ is then given by:

$$
\Gamma=2.36 \frac{\mathrm{d} E}{\mathrm{~d} x}\left[\frac{x(1-x)}{\rho V}\right]^{1 / 2}=0.2 \mathrm{meV},
$$

where $\rho=1.76 \times 10^{28} \mathrm{~m}^{-3}$ is the atomic density of diamond. This value is significantly smaller than the smallest width of bound exciton lines detected to date [51], of $\sim 1 \mathrm{meV}$, and indicates the potential still to be achieved in material quality.

\subsubsection{Dielectric properties}

When there is no absorption in a sample, the refractive index $n$ is related to the real part $\varepsilon_{1}$ of the dielectric constant by $n=\sqrt{\varepsilon_{1}}$. Measurements of the static dielectric constant, by capacitance techniques with frequencies of 1 to $10 \mathrm{kHz}$, gave $\varepsilon_{\infty}=5.70$ [100] at room temperature, consistent with the prediction of 5.72 from the $a b$ initio calculation 
of Pavone et al. [7]. For $\varepsilon_{1}=5.7, n=2.39$. At higher, optical, frequencies the refractive index increases to 2.72 for photons just below the indirect energy gap. The dependence on wavelength $\lambda$ is [105]:

$$
n^{2}=1+\frac{0.3306 \lambda^{2}}{\lambda^{2}-\lambda_{1}^{2}}+\frac{4.3356 \lambda^{2}}{\lambda^{2}-\lambda_{2}^{2}},
$$

where $\lambda_{1}=175 \mathrm{~nm}$ and $\lambda_{2}=106 \mathrm{~nm}$.

The dielectric constant varies with temperature $T$ as [106]:

$$
\varepsilon_{1}=5.701-5.35 \times 10^{-5} T+1.66 \times 10^{-7} T^{2} .
$$

Equivalently, Ruf et al. [98] have measured the refractive index up to $925 \mathrm{~K}$, and can fit the temperature variation in terms of a Bose-Einstein factor (Equation 1.20), with a single effective frequency of $711 \mathrm{~cm}^{-1}$.

One immediate practical application of the refractive index is to the reflection coefficient $R$ at normal incidence, which in the absence of absorption is $R=(n-1)^{2} /(n+1)^{2}$. The reflection coefficient increases from $17 \%$ in the infrared and visible to $21 \%$ in the near UV. At higher energies [106], near $12.6 \mathrm{eV}, R$ exceeds $50 \%$. Other aspects of the optical data have been reviewed fully by Mainwood [107], to which we add recent rationalisation of the photoelastic constants, which are important for determining strains in diamond [108]. The optical data are essential for the design of optical components fabricated from diamond. For example plano-convex lenses, of diameters of 2 to $5 \mathrm{~mm}$ and focal lengths of 3.2 to $5.2 \mathrm{~mm}$, have been made by chemical vapour deposition of diamond into spherical depressions in a substrate, followed by polishing flat the growth surface [109].

\subsection{SUMMARY}

All aspects of the basic properties of pure diamond cannot be reviewed in a short chapter. For example, we have not touched on electron transport in the bulk (which is discussed in this book by J. Isberg), surface properties, or the properties of nano-diamond. It is striking that many scientific properties of diamond have been known for considerably longer than for almost any other crystalline material, and they have played significant roles in the development of condensed matter research. However, because diamond is a simple, prototype material, its science is constantly being revisited at both the experimental and theoretical levels as new techniques become available. It will be fascinating to see how far future improvements in the growth of diamond will extend its already unique properties.

\section{REFERENCES}

[1] R.S. Balmer, I. Friel, S.M. Woollard, C.J.H. Wort, G.A. Scarsbrook, S.E. Coe, H. ElHaji, A. Kaiser, A. Denisenko, E. Kohn and J. Isberg, Phil. Trans. R. Soc. 366, 251 (2008).

[2] A. Lavoisier, Traité élémentaire de chimie, Paris Chez Cuchet 1789, reprinted Bruxelles: Culture et Civilisations, 1965. 
[3] W.H. Bragg and W.L. Bragg, Proc. R. Soc A 89, 277 (1913).

[4] A.R. Lang and G. Pang, Phil Trans. R. Soc. Lond. A 356, 1397 (1998).

[5] G.A. Slack and S.F. Bartram, J. Appl. Phys. 46, 89 (1975).

[6] C. Wild and P. Koidl, in Properties, Growth and Applications of Diamond, M.H. Nazaré and A.J. Neves (Eds), INSPEC, p. 351 (2001).

[7] P. Pavone, K. Karch, O. Schütt, W. Windl, D. Strauch, P. Giannozzi and S. Baroni, Phys. Rev. B 48, 3156 (1993).

[8] H. Holloway, H.C. Hass, M.A. Tamor, T.R. Anthony and W.F. Banholzer, Phys. Rev. B 44, 7123 (1991).

[9] T. Yamanaka, S. Morimoto and H. Kanda, Phys. Rev. B 49, 9341 (1994).

[10] P. Pavone and S. Baroni, Sol. State Commun. 90, 295 (1994).

[11] R. Vogelgesang, A.K. Ramdas, S. Rodriguez, M. Grimsditch and T.R. Anthony, Phys. Rev B 54, 3989 (1996).

[12] E.S. Zoulboulis, M. Grimsditch, A.K. Ramdas and S. Rodriguez, Phys. Rev. B 57, 2889 (1998).

[13] X. Jiang, J.V. Harzer, B. Hillebrands, Ch. Wild and P. Koidl, Appl. Phys. Lett. 59, 1055 (1991).

[14] F. Szeucs, M. Werner, R.S. Sussmann and C.S.J. Pickles, J. Appl. Phys. 86, 6010 (1999).

[15] F. Occelli, P. Loubeyre and R. Letoullec, Nature Mat. 2, 151 (2003).

[16] R. Maezono, A. Ma, M.D. Towler and R.J. Needs, Phys. Rev. Lett. 98, 025701 (2007).

[17] A.K. Ramdas, S. Rodriguez, M. Grimsditch, T.R. Anthony and W.F. Banholzer, Phys. Rev. Lett. 71, 189 (1993).

[18] C.P. Herrero and R. Ramirez, Phys. Rev. B 63, 024103 (2001).

[19] J.L. Warren, J.L. Yarnell, G. Dolling and R.A. Cowley, Phys. Rev. 158, 805 (1967).

[20] S.A. Solin and A.K. Ramdas, Phys. Rev. B 1, 1687 (1970).

[21] M.A. Washington and H.Z. Cummings, Phys. Rev. B 15, 5840 (1977).

[22] W. Windl, P. Pavone, K. Karch, O. Schütt, D. Strauch, P. Giannozzi and S. Baroni Phys. Rev. B 48, 3164 (1993).

[23] R. Vogelgesang, A.D. Alvarenga, H. Kim, A.K. Ramdas, S. Rodrigues, M. Grimsditch and T.R. Anthony, Phys. Rev. B, 58, 5408 (1998).

[24] E. Burkel, pp. 61-64 in Inelastic Scattering of X-rays with Very High Energy Resolution, Springer tracts in Modern Physics, Vol. 125, Springer, Berlin, 1991.

[25] E. Burkel, B. Dorner, Th. Illini and J. Peisl, Rev. Sci. Instrum. 60, 1671 (1989).

[26] N. Mounet and N. Marzan, Phys. Rev. B 71, 205214 (2005).

[27] A. Bosak and M. Krisch, Phys. Rev. B 72, 224305 (2005).

[28] W.L. Liu, M. Shamsa, I. Calizo and A.A. Balandin, Appl. Phys. Lett. 89, 171915 (2006).

[29] A. Debernardi, S. Baroni and E. Molinari, Phys. Rev. Lett. 75, 1819 (1995).

[30] K. Ishioka, M. Hase and M. Kitajima, Appl. Phys. Lett. 89, 231916 (2006).

[31] P.G. Klemens, Phys. Rev. 148, 845 (1966).

[32] D. Schiferi, M. Nicol, J.M. Zaug, S.K. Sharma, T.F. Cooney, T.R. Anthony and J.F. Fleischer, J. Appl. Phys. 82, 3256 (1997).

[33] P.A. Baker, Y.K. Vohra, R.S. Peterson and S.T. Weir, Appl. Phys. Lett. 83, 1734 (2003).

[34] S.S. Mitra, O. Brafman, W.B. Daniels and R.K. Crawford, Phys. Rev. B 186, 942 (1969).

[35] E. Whalley, A. Lavergne and P.T.T. Wong, Rev. Sci. Instrum. 47, 845 (1976).

[36] M. Hanfland, K. Syassen, S. Fahy, S.G. Louie and M.L. Cohen, Phys. Rev. B 31, 6896 (1985).

[37] H. Boppart, J. van Straaten and I.F. Silvera, Phys. Rev. B 32, 1423 (1985).

[38] A.F. Goncharov, I.N. Makarenko and S.M. Stishov, JETP Lett. 41, 184 (1985).

[39] M.S. Liu, L.A. Bursill and S. Prawer, Phys. Rev. B 61, 3391 (2000).

[40] E.S. Zouboulis and M. Grimsditch, Phys. Rev. B 43, 12490 (1991).

[41] H. Herschen and M.A. Capelli, Phys. Rev. B 43, 12490 (1991).

[42] M.H. Grimsditch, E. Anastassakis and M. Cardona, Phys. Rev. B 18, 901 (1978). 
[43] E. Anastassakis, A. Cantarero and M. Cardona, Phys. Rev. B 41, 7529 (1990).

[44] Y. Akahama and H. Kawamura, J. Appl. Phys. 98, 083523 (2005).

[45] J.F. Angress, C. Cooke and A.J. Maiden, J. Phys. C 1, 1769 (1968).

[46] J. Wagner, C. Wild, W. Mullersebert and P. Koidl, Appl. Phys. Lett. 61, 1284 (1992).

[47] J. Wagner, C. Wild and P. Koidl, Appl. Phys. Lett. 59, 779 (1991).

[48] P.J. Dean, E.C. Lightowlers and D.R. Wight, Phys. Rev. 140 A352 (1965).

[49] G. Davies, J. Phys. Chem. Solids 31, 883 (1970).

[50] A.T. Collins, S.C. Lawson, G. Davies and H. Kanda, Phys. Rev. Lett. 65, 891 (1990).

[51] S.J. Sharp, A.T. Collins, G. Davies and G.S. Joyce, J. Phys. C: Condens. Matter 9, L451 (1997).

[52] C.A. Klein, T.M. Hartnett and C.J. Robinson, Phys. Rev. B 45, 12854 (1992).

[53] B.J. Parsons, Proc. R. Soc. Lond. A 352, 397 (1976).

[54] C. Piccirillo, G. Davies, A. Mainwood, S. Scarle, C.M. Penchina, T.P. Mollart, K.L. Lewis, M. Nesládek, Z. Remes and C.S.J. Pickles, J. Appl. Phys. 92, 756 (2002).

[55] J. Menéndez and M. Cardona, Phys. Rev. B 29, 2051 (1984).

[56] R.M. Chrenko, J. Appl. Phys. 63, 5873 (1988).

[57] K.C. Haas, M.A. Tamor, T.R. Anthony and W.F. Banholzer, Phys. Rev. B 45, 7171 (1992).

[58] J. Spitzer, P. Etchegoin, M. Cardona, T.R. Anthony and W.F. Banholzer, Solid State Commun. 88509 (1993).

[59] T. Ruf, M. Cardona, H. Sternschulte, S. Wahl, K. Thonke, R. Sauer, P. Pavone and T.R. Anthony, Solid State Commun. 105, 311 (1998).

[60] A.C. Victor, J. Chem. Phys. 36, 1903 (1962).

[61] W. DeSorbo, J. Chem. Phys. 21, 1903 (1962).

[62] J.E. Desnoyers and J.A. Morrison, Phil. Mag. 3, 42 (1958).

[63] H.E. Weber, Philos. Mag. 49, 161 (1875).

[64] A. Einstein, Ann. Phys. 22, 180 (1907).

[65] J.E. Graebner, Diamond Rel. Mater. 5, 1366 (1996).

[66] M. Sanati, S.K. Estreicher and M. Cardona, Solid State Commun. 131, 229 (2004); M. Cardona, R.K. Kremer, M. Sanati, S.K. Estreicher and T.R. Anthony, Solid State Commun. 133, 465 (2005).

[67] M. Cardona, T.A. Meyer and M.L.W. Thewalt, Phys. Rev. Lett. 92, 196403 (2004).

[68] J. Calloway, Phys. Rev. 113, 1046 (1959).

[69] M. Reichling and J. Hartmann, in Properties, Growth and Applications of Diamond, M.H. Nazaré and A.J. Neves (Eds), INSPEC, p. 55 (2001).

[70] J.W. Vandersande, Phys. Rev. B 13, 4560 (1976).

[71] R. Berman, P.R.W. Hudson and M. Martinez, J. Phys. C. Solid State Phys. 8. L430 (1975).

[72] E.A. Burgemeister, Physica B \& C 93, 165 (1978).

[73] E.A. Burgemeister, C.A. Ammerlaan and G. Davies, J. Phys. C: Solid St. Phys. 13, L691 (1980).

[74] L. Wei, P.K. Kuo, R.L. Thomas, T.R. Anthony and W.F. Banholzer, Phys. Rev. Lett. 70, 3764 (1993).

[75] J.E. Graebner, S. Jin, G.W. Kammlott, J.A. Herb and C.F. Gardinier, Nature 359, 401 (1992).

[76] J. Hartmann, M. Costello and M. Reichling, Phys. Rev. Lett. 80, 117 (1998).

[77] D.J. Twitchen, C.S.J. Pickles, S.E. Coe, R.S. Sussmann and C.E. Hall, Diamond Rel. Mater. 10, 731 (2001).

[78] E. Woerner, J. Wagner, W. Mueller-Sebert, C. Wild and P. Koidl, Appl. Phys. Lett. 68, 1482 (1996).

[79] K.I. Belay, Z.Y. Etzel, D.G. Onn and T.R. Anthony, J. Appl. Phys. 79, 8336 (1996).

[80] M. Cardona, T. Ruf and J. Serrano, Phys. Rev. Lett. 86, 3923 (2001).

[81] C.D. Clark, P.J. Dean and P.V. Harris, Proc. R. Soc. Lond. A 277, 312 (1964).

[82] H.R. Philipp and E.A. Taft, Phys. Rev. A136, 1445 (1964). 
[83] R.A. Roberts and W.C. Walker, Phys. Rev. 161, 730 (1967).

[84] A.D. Papadopoulos and E. Anastassakis, Phys. Rev. B 43, 5090 (1991).

[85] I. Jiménez, L.J. Terminello, D.G.J. Sutherland, J.A. Carlisle, E.L. Shirley and F.J. Himpsel, Phys. Rev. B 56, 7215 (1997).

[86] M. Hybertsen and S.G. Louie, Phys. Rev. B 34, 5390 (1986).

[87] M. Rohlfing, P. Kruger and J. Pollmann, Phys. Rev. B 48, 17791 (1993).

[88] M. Willatzen, M. Cardona and N.E. Christensen, Phys. Rev. B 50, 18054 (1994).

[89] F. Nava, C. Canali, C. Jacobini, L. Reggiani and S.F. Kozlov, Solid State Commun. 33, 475 (1980).

[90] E. Gheeraert, N. Casanova, S. Koizumi, T. Teraji and H. Kanda, Diamond Rel. Mater. 10, 444 (2001).

[91] R. Sauer, H. Sternschulte, S. Wahl, K. Thonke and T.R. Anthony, Phys. Rev. Lett. 84, $4172(2000)$.

[92] A. Onodera, M. Hasegawa, K. Furuno, M. Kobayashi and Y. Nisida, Phys. Rev. B 44, 12176 (1991).

[93] F.J. Himpsel, J.F. van der Veen and D.E. Eastman, Phys. Rev. B 22, 1967 (1980).

[94] A.S. Barnard, S.P. Russo and I.K. Snook, Phys. Rev. B 69, 235407 (2003).

[95] P.E. van Camp, V.E. Van Doren and J.T. Devreese, Phys. Rev. B 34, 1314 (1986).

[96] S. Zollner, M. Cardona and S. Gopalan, Phys. Rev. B 45, 3376 (1992).

[97] K.P. O’Donnell and X. Chen, Appl. Phys. Lett. 58, 2924 (1991).

[98] T. Ruf, M. Cardona, C. Pickles and R. Sussmann, Phys. Rev. B 62, 16578 (2000).

[99] S. Logothetidis, J. Petalas, H.M. Polatoglou and D. Fuchs, Phys. Rev. B 46, 4483 (1992).

[100] J. Fontanella, R.L. Johnston, J.H. Colwell and C. Andeen, Appl. Opt. 16, 2949 (1977).

[101] A. Kujii, K. Takiyama, R. Maki and T. Fujita, J. Lumin. 94, 355 (2001).

[102] R. Shimano, M. Nagai, K. Horiuch and M. Kuwata-Gonokami, Phys. Rev. Lett. 88, 057404 (2002).

[103] K. Thonke, R. Schielsing, N. Teofilov, H. Zacharias, R. Sauer, A.M. Zaitsev, H. Kanda and T.R. Anthony, Diamond Rel. Mater. 9, 428 (2000).

[104] R. Sauer, N. Teofilov and K. Thonke, Diamond Rel. Mater. 13, 691 (2004).

[105] F. Peter, Z. Phys. 15, 358 (1923).

[106] W.C. Walker and J. Osantowski, Phys. Rev. 134, A153 (1964).

[107] A. Mainwood, Optical constants of diamond, in Properties and Growth of Diamond, G. Davies (Ed.), Electronic Materials Information Service Data Reviews Series, INSPEC, London, 1994.

[108] L.S. Hounsome, R. Jones, M.J. Shaw and P.R. Briddon, Phys. Stat. Sol. (a), 203, 3088 (2006).

[109] E. Woerner, C. Wild, W. Mueller-Sebert and P. Koidl, Diamond Rel. Mater. 10557 (2001). 